\title{
PELTIER EFFECT APPLIED TO THE DESIGN AND REALIZATION OF A NEW MASS FLOW SENSOR
}

\author{
M. RAHMOUN ${ }^{a}, b, *$, A. EL HASSANI ${ }^{b}$, D. LECLERQ ${ }^{c}$ \\ and E. BENDADA ${ }^{\mathrm{a}}$ \\ ${ }^{a}$ Laboratoire de Micro-électronique et de Capteurs et Instrumentation, \\ Dépt. De Physique, Université My Ismaïl Faculté des Sciences et Techniques \\ Errachidia Maroc; \\ ${ }^{\mathrm{b}}$ Laboratoire d'Electronique et de Traitement de l'Information, Université \\ My Ismaïl Faculté des Sciences de Meknès Maroc; \\ ${ }^{\mathrm{c}}$ Laboratoire Capteurs et Instrumentation, Université des Sciences \\ et Technologies de Lille France
}

(Received 20 May 1999; In final form 20 July 1999)

The present paper deals with design and realization of a new mass flow sensor using the Peltier effect. The sensor, shaped as a bimetallic circuit includes two continuous parallel strips coated with a great deal of metal plated spots. In such a device, one track performs as a classical thermoelectrical circuitry whose both plated and uncoated parts provide the thermopile junctions. The other strip is subjected to electrical current so as to generate numerous small thermal gradients owing to the Peltier effect. Then, the resulting differences in temperature induce a Seebeck e.m.f. detected by the other strip acting as a receiver. The thermal coupling between transmitter and receiver tracks depends on many variation of the surrounding environment heat transfer coefficient. Therefore, such a device allows us to detect any shift in physical properties related to the apparent thermal conductivity. In special case of a steady state fluid, the induced e.m.f. in the receiving track hinges on the thermal conductivity. When the fluid is in relative motion along the sensor, the velocity can be read out as a function of voltage as an application, the sensor is placed into a tube conducting a fluid flow, in order to design a new mass flowmeter.

Keywords: Thermoelectricity; sensor; flowmeter

\footnotetext{
*Corresponding author.
} 


\section{INTRODUCTION}

The hot wire anemometer has been used for many years as a research tool in fluid mechanics [1]. A hot wire anemometer usually refers to the use of small electrically heated element placed in a fluid with the aim of measuring a property of that medium. Normally, the property being measured is the velocity. Since these elements are sensitive to heat transfer between the element and its environment, temperature and composition changes can also be measured. Film type sensors have been used for a great variety of measurements such as turbulent levels in a wind tunnel, flow patterns around models, etc. Monolithic silicon thermal flow sensors for measuring the velocity of gas or liquid have appeared in the literature [2] and can be used as the hot wire for mass flow measurements in a pipe. In this paper a new sensor based on the Peltier [3,4] effect is presented which is particularly useful for the purpose of measuring mass flow in a pipe.

\section{OPERATING PRINCIPLE}

The sensing element is basically a thermopile (Fig. 1) compressing a thin continuous strip foil of low conductivity overlaid with numerous electroplated patches of copper coatings of high conductivity. It has recognized [6] that when the plated portions are located in a

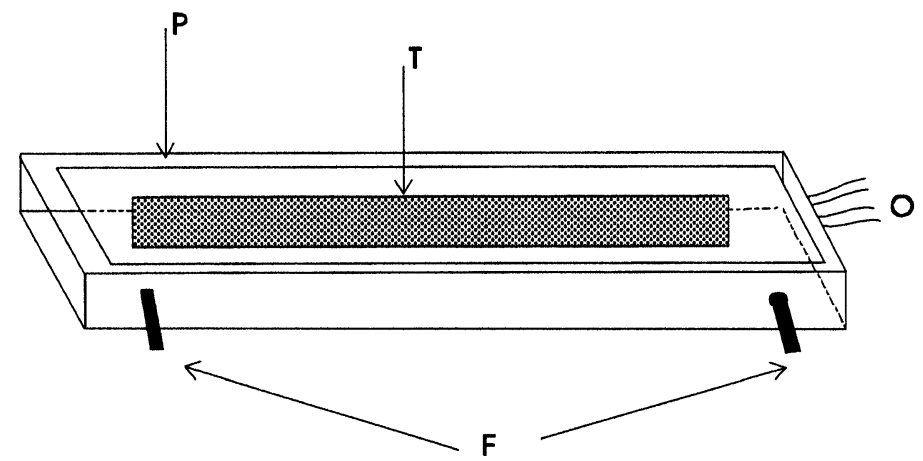

FIGURE 1 Schematic view of the cell used $P$, pyrex tube; $F$, fluid; $T$, thermoelectric sensor; $o$, output coaxial cable. 
temperature gradient between a heat source region and a heat sink region a number of thermocouples junctions operating at the coating boundaries are interconnected in series. If such a thermopile, immersed in a fluid, is operating without electric current flowing through it, the junction points are kept at the fluid temperature. If the fluid temperature is uniformly distributed in space, the temperature difference between each couple of junction points is equal to zero and so is the output voltage measured between the ends of the continuous foil strip. Let us now assume that a very low current has been flowing through the circuit, generating the Joule effect for a sufficiently long time to reach thermal equilibrium. On account of the difference between the conductivities of the surimposed metallic materials most of the current will pass through the electrodes placed in parallel with the poorly conducting support (Fig. 2). Since the part of the current that enters and leaves the poorly conducting continuous foil strip passes near the junction points and near the electrode ends, heating and cooling effect by the Peltier effect (as low as $12 \mu \mathrm{W}$ for a current of $1 \mathrm{~mA}$ ) alternately localized at the junction points take place.
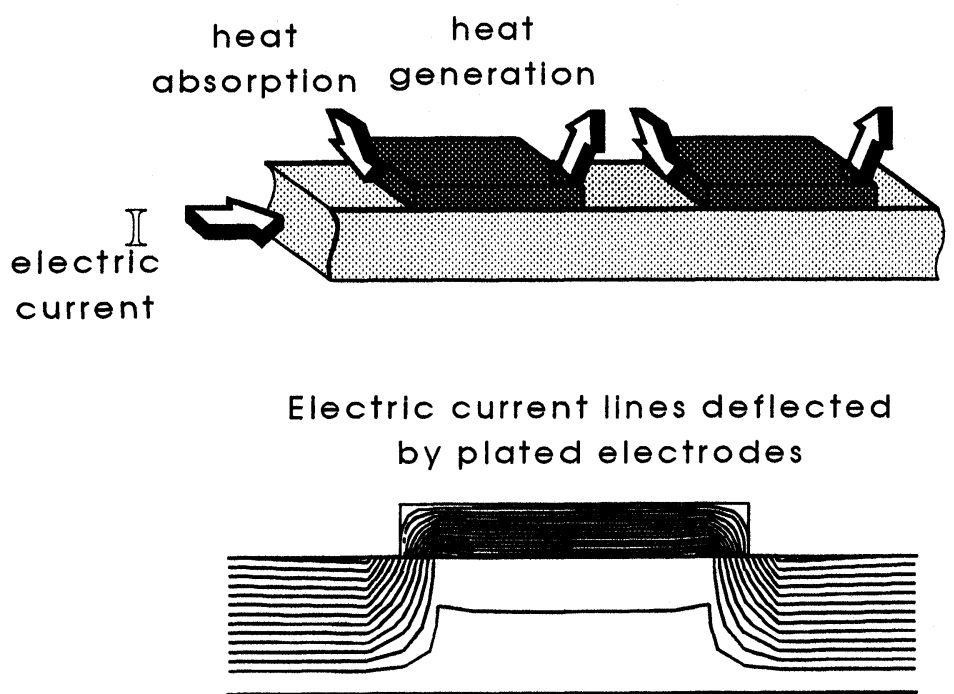

FIGURE 2 (a) Heat generation and absorption with Peltier effect on thermoelectric junctions. (b) Electric current lines deflected by plated electrodes close to the thermoelectric junctions. 
At the initiation of the electric current, temperature difference $\Delta T$ are then developed between each couple of junction points giving thus a Peltier e.m.f. depending directly on the thermal resistance between the junction points. Consequently, any change in the thermal resistance between the junction points is directly converted into a proportional change in the Peltier voltage. Since the thermal resistance between two consecutive junction points is directly influenced by the fluid acting in parallel with the thermopile material, any change in the fluid heat transmission characteristic will be directly converted into electric voltage which may be used to measure it. To perform measurements based on the Peltier effect, it is essential that any change in the heat transmission characteristic of the fluid can be operate a change in the part of Peltier heat conducted in the thermopile material from the hot to the cold junction. In other words, any decrease in the heat transmission characteristic of the fluid will decreased the part of Peltier heat removed through convection into the fluid from the junction points. Moreover, since heat generation by the Peltier effect at the junction points is maintained at a constant rate, a greater part of Peltier heat is conducted from the hot to the cold junction in the thermopile materials, thus developing a greater Peltier voltage. Conversely, any increase in the fluid heat transmission characteristic will decrease the part of Peltier heat conducted through the thermopile materials and thus give a proportional decreased in the Peltier voltage generated around the thermopile circuit. In order to obtain high sensitivity to the fluid heat transmission characteristic, which may be increased or decreased around a reference value, it is desirable to select the dimension and shape factor of the plated electrodes in such a way that the part of Peltier heat conducted from the hot to the cold junction through the thermopile is nearly equal to the part removed through conduction and convection into the fluid in its reference state. Under such circumstances, the thermal resistance of the thermopile between two consecutive junction points is matched to that of the fluid between the same points.

The problem then remaining is to find a method to measure the Peltier voltage occurring in the strip. When current I flows through the strip, the actual output voltage is equal to the sum of the ohmic drop due to the circuit electric resistance plus the Peltier voltage.

On account of the electrical resistance temperature dependence, the ohmic drop changes which are in fact one or two orders of magnitude 


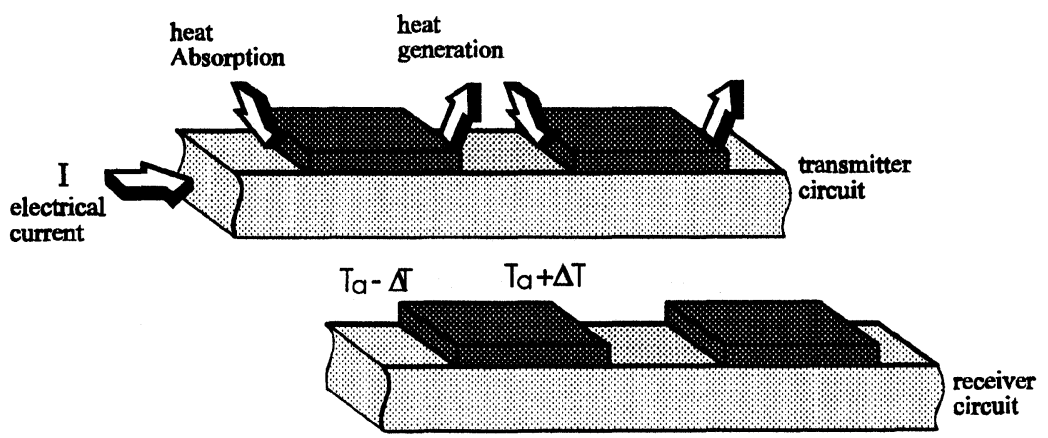

FIGURE 3 Section of the thermoelectric sensor.

above the Peltier voltage to be measured, renders its direct extraction from the output voltage quite impossible.

In order to measure the small Peltier voltage independentely of the ohmic drop influenced by the temperature of the circuit, the sensor includes two continuous parallel tracks, one track (transmitter) is crossed by an electrical current in order to generate temperature gradients between its junction points. The other parallel track (receiver) used to detect these temperature gradients (Fig. 3) and generates a voltage proportional to the temperature differences between junction points.

A sequence of data points, which represents the Peltier voltage, is thus obtained. It is the sum of the temperature differences taking place on the bimetallic sensor as it varies with time. The problem that remains now is to relate that Peltier voltage to the mass flow rate for a fluid flow.

\section{EXPERIMENTAL RESULTS}

The experimental part presents a whole set of results which are produced by a new sensor based on the coupling between transmitter and receiver tracks. We have realized two parallel bimetallic printed circuit (copper-constantan) on Kapton support (Fig. 4). Each bimetallic track of width $0.2 \mathrm{~mm}$ and length $800 \mathrm{~mm}$ is folded up in order to be placed into a pipe. The junction points are localized on the line borders of the plated electrodes and the spacing between them is $3 \mathrm{~mm}$. The sensor thus produced is dimensioned in order to be placed in a 


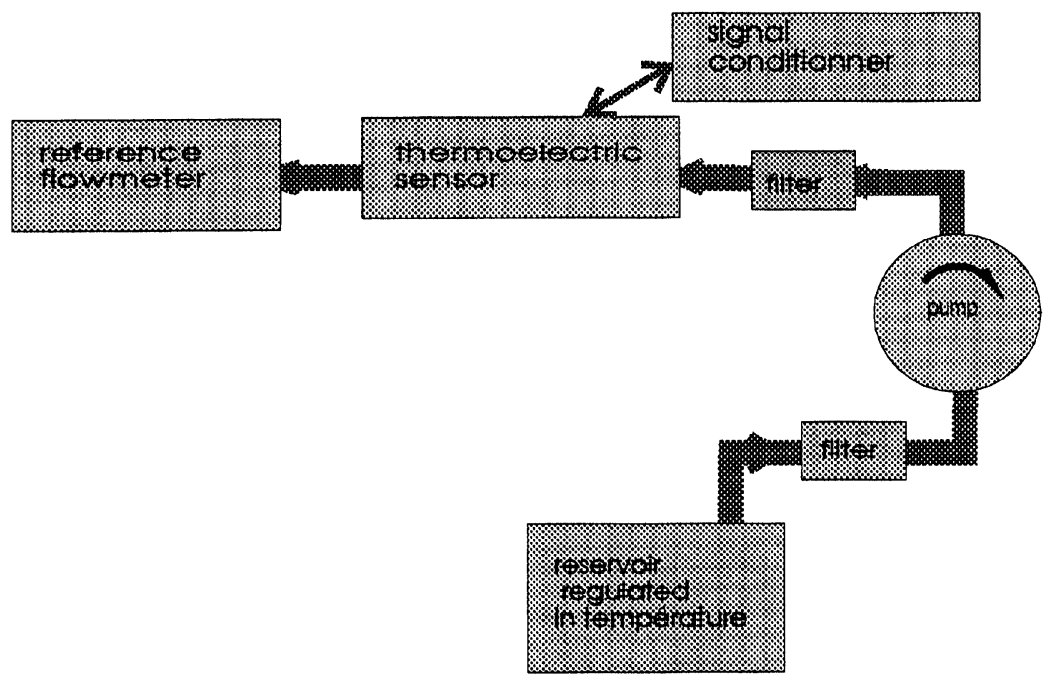

FIGURE 4 Apparatus used to obtain a steady mass flow rate.

conduct, where a fluid of known thermoelectric properties may circulate and where the fluid velocity changes may be controlled.

The experimental device used to obtain a steady mass flow rate is shown in Figure 5.

\subsection{Measurement of Thermal Gradients Caused by the Electrical Current}

When the electric current has been flowing through the transmitting track, the temperature gradients generated by Peltier effect are localized on the junction points. The temperature differences are directly converted into a proportional Seebeck voltage.

The variation of this voltage against a current flowing through the transmitting track is represented in Figure 6 for a fluid (water).

The calibration curve is linear and are proportional to the dissipated Peltier power in the transmitting circuit. For a determined current intensity, the temperature differences between junction points are more significant when the fluid conductivity is low. This is due to the influence of the fluid conductivity on the thermal resistance between the junction points. 


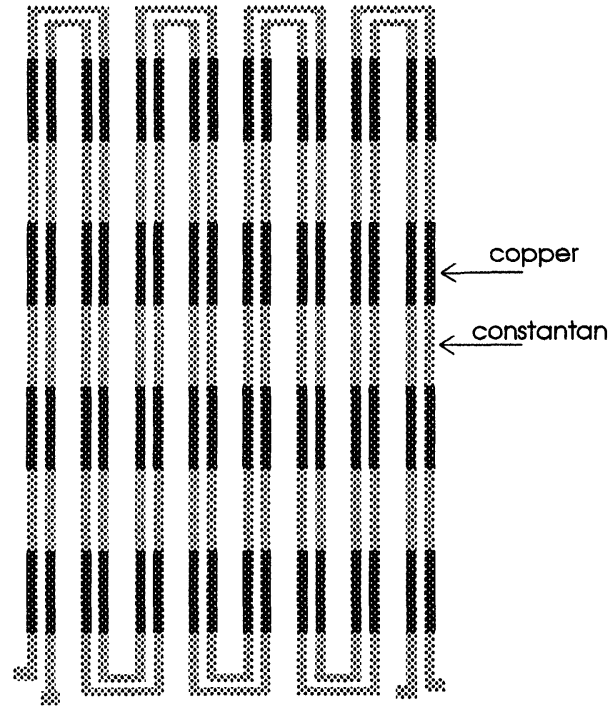

FIGURE 5 Thermoelectric sensor includes two parallel strips.

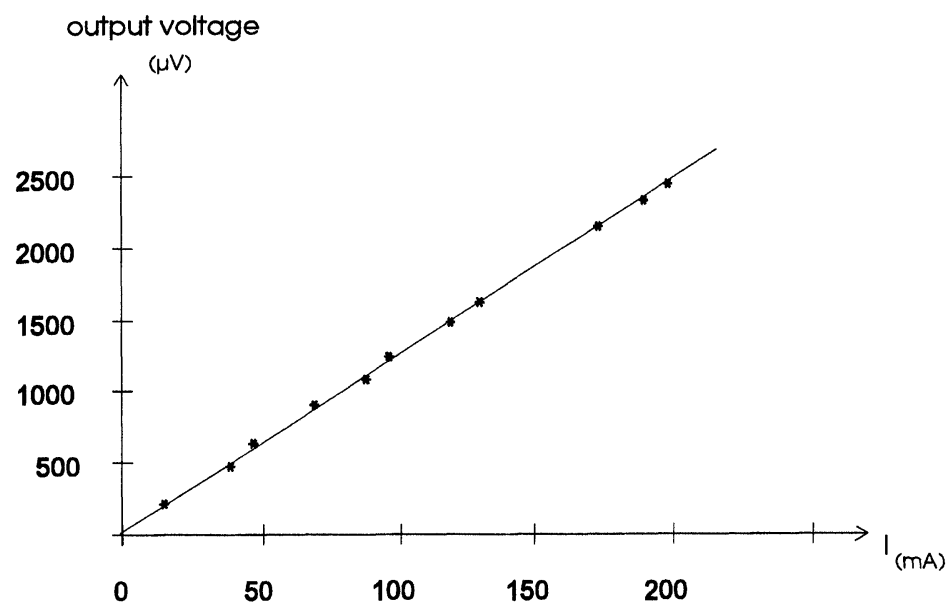

FIGURE 6 Variation the output voltage against the electric current.

\subsection{Application for Measuring Mass Flow}

The sensor was mounted on the center of a rectangular flow tube. In order to take flow rate measurement, the experimental procedure 
which we have adapted is:

- pass a constant current in the transmitting track so that thermal gradients are created and,

- measure the detected e.m.f. by the receiving track as a function of the flow rate.

In the absence of the fluid, the output voltage $V_{0}$ takes a reference values (zero flow value) depending only on the fluid thermal conductivity. When a flow is induced, there is a spacial change in the convective heat flow from the lateral surface which depends on the thermal boundary condition on the material surface. Thus a change in the temperature differences between the junction points gives a change in Seebeck voltage. By measuring the variation $\Delta V=V_{0}-V$, the mass flow measurement can be obtained. In order to not take account the dissipated Joule power $P$ in the transmitting track, we trace the variation $\Delta V / P$.

The calibration curves (Fig. 7) are non linear with maximum sensitivity at the low flow rates decreasing towards high flow rates. A significant advantage of the non linear output characteristic is the ability to make measurements over a wide range of flow rates while maintaining a nearly constant "percentage of reading" accuracy. By changing the fluid pressure in the sensor over the range $(0.01-1 \mathrm{bar})$

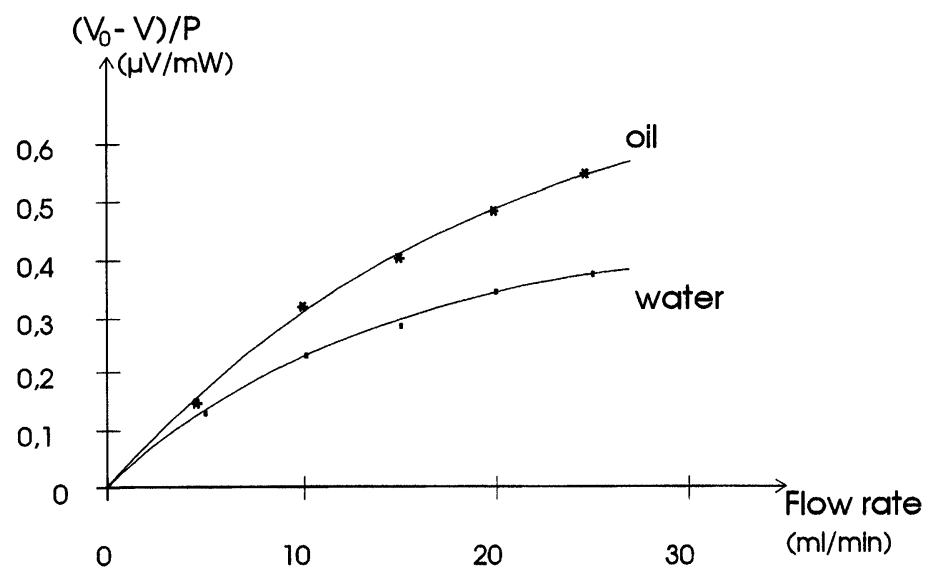

FIGURE 7 Variation the output voltage against mass flow rate: Conductivity of oil is $0.169 \mathrm{~W} / \mathrm{m} \cdot \mathrm{K}$; Conductivity of water is $0.569 \mathrm{~W} / \mathrm{m} \cdot \mathrm{K}$. 
while maintaining constant the flow rate measured by the reference flowmeter, it was shown that the output as well the calibration curves were interchanged and not dependent on the fluid pressure variations, it clearly appears that the fundamental measured variable is the mass flow rate. The volumetric flow may also be obtained wherever density is constant.

\subsection{Dynamic Response}

Another important characteristic of a flow sensor is its dynamic response. This was measured using a system in which the fluid flow could be controlled so that the flow originally equal to zero was then abruptly established through the Peltier sensor. The response time defined as the time to reach full scale is approximately $26 \mathrm{~ms}$. Since electronic circuitry time response was lower than $1 \mathrm{~ms}$, this suggested that the measured response was due to the sensor. The inherent speed of Peltier element is very high since it responds to changes in heat transmission characteristic of the fluid and not to temperature itself.

\section{CONCLUSION}

This study demonstrates that the Peltier sensor is very sensitive and versatile flow sensor, giving a zero flow output depending on the fluid thermal conductivity. It suggests that the prospect for highly reproducible manufacture of this device is good. The reproducible at any given flow level suggests that the Peltier sensor has interesting applications as a transfer standard in the detailed calibration of flow sensor against a well defined standard.

\section{References}

[1] Bruun, H. H., Khan, M. A., Al Kaijien, H. H. and Farad, A. A. (1988). Velocity calibration relationships for hot wire anemometry, J. Phys. E: Sci. Instrum., 21, $225-232$.

[2] Oudheusden, B. W. (1990). Silicon thermal flow sensor with a two dimensional direction sensitivity, Meas. Sci. Technol., 1, 565-575.

[3] Leclerq, D., Wattiau, F. and Thery, P. (1989). Procédé et dispositif utilisant les effets thermoélectriques pour la mesure d'une grandeur physique dont la variation est apte à modifier les propriétés thermophysiques d'un milieu French Patent 8916809. 
[4] Rahmoun, M. (1994). Conception et réalisation d'un dispositif à effet Peltier adapté à la mesure des faibles débit de liquide. Thèse de l'Université de Lille France.

[5] Hladik, J. (1990). Metrologie des propriétés thermophysiques des matériaux, Edition Masson.

[6] Knibble, P. G., An accurate instrument for fast thermal conductivity and thermal diffusitivity measurements at elevated temperature and pressure, J. Phys. E: Sci. Instrum., 20, 1205-11. 

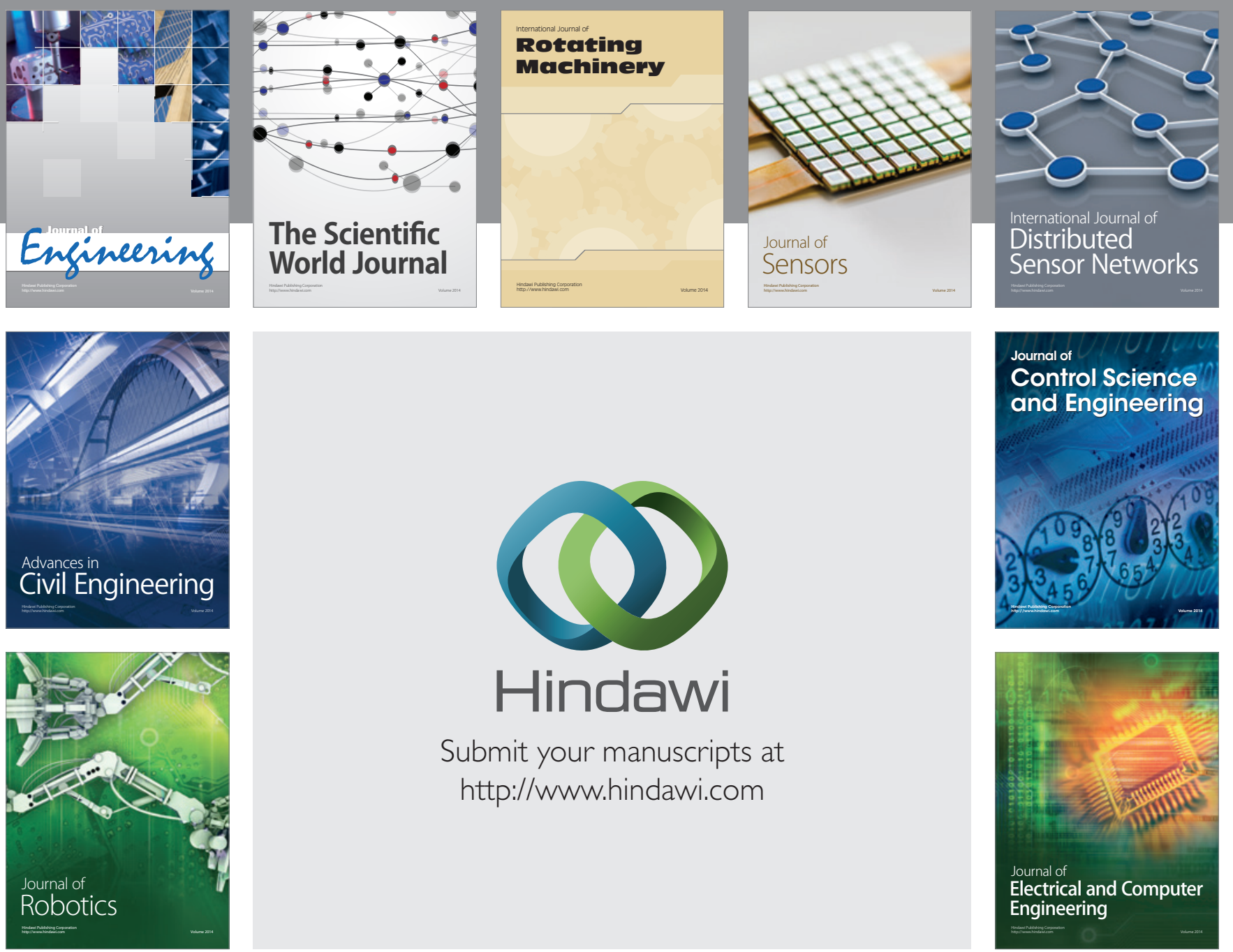

Submit your manuscripts at

http://www.hindawi.com
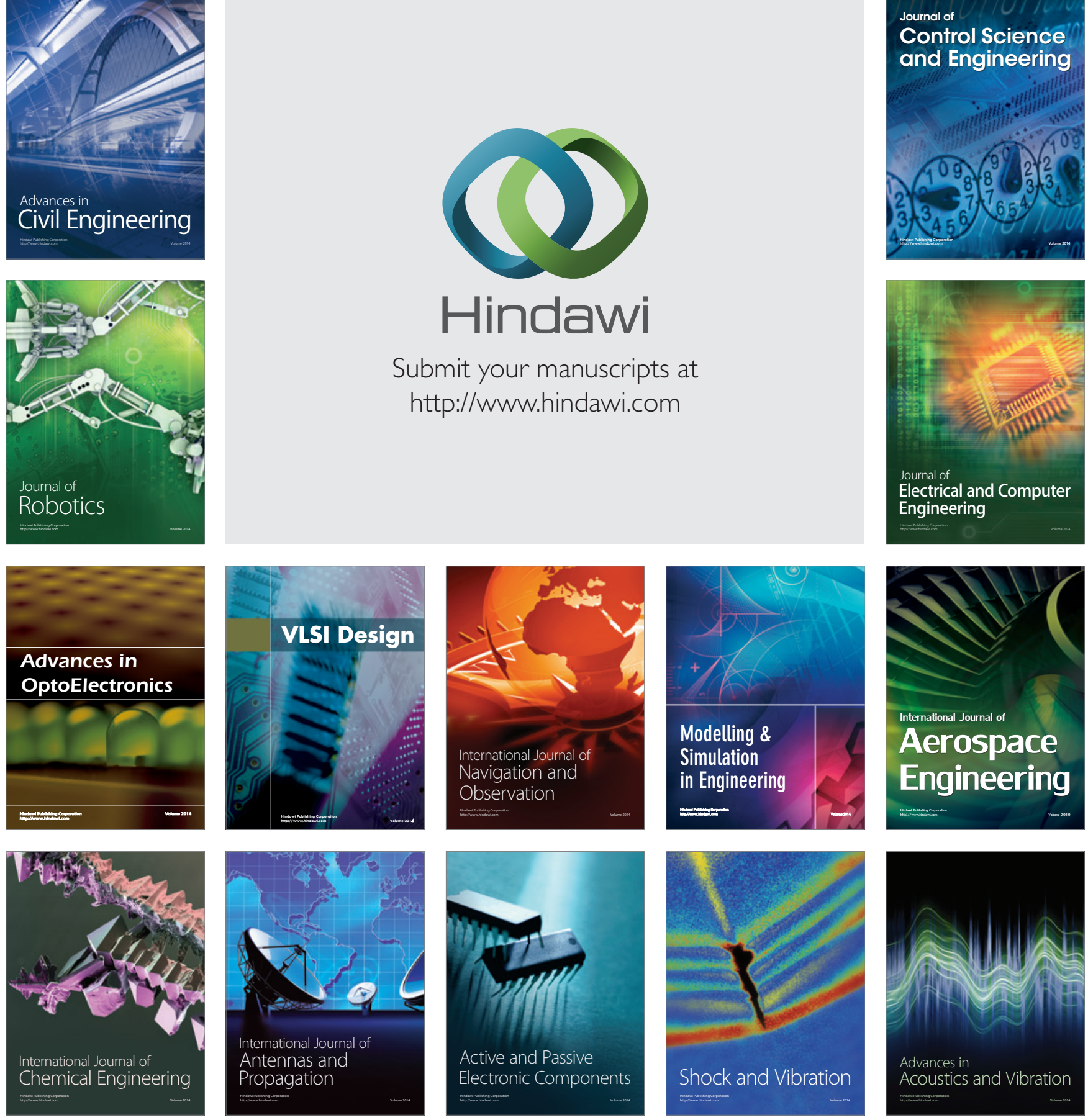